\title{
Four-jet production via double parton scattering in $p A$ collisions at the LHC
}

\author{
Boris Blok $^{1, *}$ and Federico Alberto Ceccopieri ${ }^{1,2, \dagger}$ \\ ${ }^{1}$ Department of Physics, Technion, Israel Institute of Technology, Haifa 32000, Israel \\ ${ }^{2}$ IFPA, Université de Liège, B4000 Liège, Belgium
}

(Received 1 April 2020; accepted 14 May 2020; published 26 May 2020)

\begin{abstract}
We present predictions for the double parton scattering (DPS) four-jet production cross sections in $p A$ collisions at the LHC. Relying on the experimental capabilities to correlate centrality with impact parameter $B$ of the proton-nucleus collision, we discuss a strategy to extract the double parton scattering contributions in $p A$ collisions, which gives direct access to double parton distribution in the nucleon. We show that the production cross sections via DPS of four jets, out of which two may be light- or heavy-quark jets, are large enough to allow the method to be used already with data accumulated in the $2016 \mathrm{pA}$ run.
\end{abstract}

DOI: 10.1103/PhysRevD.101.094029

\section{INTRODUCTION}

The flux of incoming partons in hadron-induced reactions increases with the collision energy so that multiple parton interactions (MPIs) take place, in both $p p$ and $p A$ collisions. The study of MPIs started in the 1980s in the Tevatron era [1-3], both experimentally and theoretically. Recently, significant progress was achieved in the study of MPIs, in particular of double parton scattering (DPS). From the theoretical point of view, a new self-consistent perturbative QCD (pQCD) based formalism was developed for both $p p$ [4-13] and $p A$ DPS collisions [14] (see Ref. [15] for recent reviews). Recent observations of double open charm [16-19] and same-sign $W W(s s W W$ ) production [20] clearly show the existence of DPS interactions in $p p$ collisions.

The MPI interactions play a major role in the underlying event (UE) and thus are taken into account in all Monte Carlo generators developed for the LHC [21,22]. On the other hand, the study of DPS will lead to understanding of two parton correlations in the nucleon. In particular, the DPS cross sections involve new nonperturbative two-body quantities, the so-called two particle generalized parton distribution functions $\left({ }_{2}\right.$ GPDs), which encode novel features of the nonperturbative nucleon structure. Such distributions have the potential to unveil two-parton correlations in the nucleon structure $[23,24]$ and

\footnotetext{
*blok@physics.technion.ac.il

†ederico.ceccopieri@hotmail.it
}

Published by the American Physical Society under the terms of the Creative Commons Attribution 4.0 International license. Further distribution of this work must maintain attribution to the author(s) and the published article's title, journal citation, and DOI. Funded by SCOAP ${ }^{3}$. to give access to information complementary with respect to the one encoded in nucleon one-body distributions.

The study of MPI and in particular of the DPS reactions in $p A$ collisions is important for our understanding of MPI in $p p$ collisions, and it constitutes a benchmark of the theoretical formalism available for these processes. On the other hand, the MPI in $p A$ collisions may play an important role in the UE and high multiplicity events in $p A$ collisions. Moreover, it was argued in Ref. [14] that they are directly related to longitudinal parton correlations in the nucleon.

The theory of MPI and in particular DPS in $p A$ collisions was first developed in Ref. [25], in which it was shown that there are two DPS contributions at work in such a case. First, there is the so-called DPS1 contribution, depicted in the left panel of Fig. 1, in which two partons from the incoming nucleon interact with two partons in the target nucleon in the nucleus, making such a process formally identical to DPS in the $p p$ collisions. Next, there is a new type of contribution, depicted in the right panel of Fig. 1, which we refer to as DPS2, in which two partons from the incoming nucleon interact with two partons from distinct nucleons in the target nucleus located at the same impact parameter. Such a contribution is parametrically enhanced by a factor $A^{1 / 3}$ over the DPS 1 contribution, $A$ being the atomic number of the nucleus.

In the recent past, a number of theoretical studies which focus on the study of DPS contributions in $p A$ collisions have appeared [14,26-30]. However, although many interesting theoretical studies of DPS2 have been performed recently, the problem that remains is how to observe DPS2 experimentally. The main issue is obviously the large single parton scattering (SPS) (leading twist) background in such processes, which makes the observation of the DPS contributions, which are next to leading twist phenomena, a rather complicated task. 


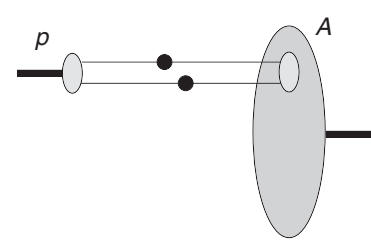

(a)

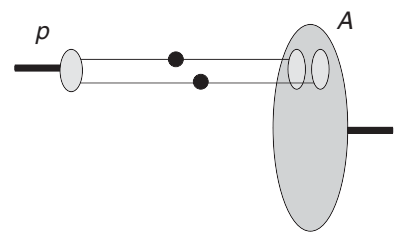

(b)

FIG. 1. Pictorial representation of DPS process in $p A$ collisions via a) DPS1 and b) DPS2 mechanisms. The light gray blobs indicate nucleons, darker gray ones indicate the nucleus, and black ones indicated the hard interactions.

Recently, however, a new method was suggested in Ref. [31] and allows to separate DPS2 from the leading twist (and DPS1) contributions. The method exploits the different dependence on the impact parameter $B$ on the various contributions to the $p A$ cross section for a given final state: while the SPS and DPS1 contributions are proportional to the nuclear thickness function $T(B)$, the DPS2 one is proportional to the square of $T(B)$. Therefore, the cross section producing a given final state can be schematically written as [31]

$\frac{d^{2} \sigma_{p A}}{d^{2} B}=\left(\sigma_{p A}^{L T}+\sigma_{p A}^{D P S 1}\right) \frac{T(B)}{A}+\sigma_{p A}^{D P S 2} \frac{T^{2}(B)}{\int d^{2} B T^{2}(B)}$,

where $T(B)$ is normalized to the atomic number $A$ of the nucleus. This approach was used in Ref. [31] to study twodijets processes and in Ref. [32] to study processes involving the associated production of electroweak bosons and jets in $p A$ collisions.

The latter strategy exploits the experimental capabilities to accurately relate centrality with the impact parameter $B$ of the $p A$ collisions. The procedure for the determination of centrality in $p A$ collisions was developed, i.e., by ATLAS [33]. It makes use of the measurement of the transverse energy $E_{T}$ deposited in the pseudorapidity interval $-3.2 \geq$ $\eta \geq-4.9$ (i.e., along the nucleus direction) as a measure of centrality. It was shown in Ref. [34] that $E_{T}$ in this kinematics is not sensitive to production of hadrons at forward rapidities. The $E_{T}$ distribution as a function of the number of collisions $\nu$ (and thus on the impact parameter $B$ ) is presented in Refs. [33-35] (see also the related discussion in Ref. [31]).

The purpose of the present paper is to continue the research started in those works and pursue the emergence of DPS2 contribution in the four-jet final state. Indeed, the observation of DPS in $p A$ collisions faces two main challenges: the first one, in common with DPS studies in $p p$ collisions, is tackling the large SPS background; the second one is given by the limited integrated luminosity accumulated in short $p A$ runs, which is several orders of magnitude lower than the one accumulated in $p p$ collisions. Therefore, the obvious question is whether the number of observed DPS events is sufficient to overcome the systematic inaccuracy due to the large SPS background. Such a question was studied, for example, in Ref. [32], in which we found that it is possible to separate SPS and DPS2 backgrounds for the $W j j$ final state.

The purpose of this paper is to investigate the possibility of isolating the DPS2 contribution within the multijet final state and the necessary kinematic constraints. We shall calculate the cross sections as a function of impact parameter $B$ of the $p A$ collision, for its various components in both the four-jet $(4 j)$ and two $b$-jet plus two light jets $(2 b 2 j)$ final states, and estimate the sensitivity to the DPS mechanisms for the considered final states. We shall see that both these final states are the "golden plate" channel for the observation of the DPS2 mechanism.

The paper is organised as follows. In Sec. II, we review the theoretical formalism and the setup for our calculations. In Secs. III and IV, we analyze and discuss the results in the $4 j$ and $2 b 2 j$ final states, respectively. We summarize our results in the conclusions.

\section{THEORETICAL FRAMEWORK}

The cross section for the production of final states $C$ and $D$ in $p A$ collisions via double parton scattering can be written as the convolution of the ${ }_{2}$ GPDs of the proton and the nucleus, $G_{p}$ and $G_{A}$, respectively [14,25]:

$$
\begin{aligned}
\frac{d \sigma_{D P S}^{C D}}{d \Omega_{C} d \Omega_{D}}= & \frac{m}{2} \int \frac{d^{2} \vec{\Delta}}{(2 \pi)^{2}} \frac{d \hat{\sigma}_{i k}^{C}\left(x_{1}, x_{3}\right)}{d \Omega_{C}} \frac{d \hat{\sigma}_{j l}^{D}\left(x_{2}, x_{4}\right)}{d \Omega_{D}} \\
& \times G_{p}^{i j}\left(x_{1}, x_{2}, \vec{\Delta}\right) G_{A}^{k l}\left(x_{3}, x_{4},-\vec{\Delta}\right) .
\end{aligned}
$$

${ }_{2}$ GPDs depend on the transverse momentum imbalance momentum $\vec{\Delta}$. The structure and relative weight of different contributions to the nuclei ${ }_{2}$ GPDs were studied in detail in Ref. [14], in which it was shown that only two contributions survive: the one that corresponds to DPS1 mechanism and an other one corresponding to DPS2.

Since our analysis will especially deal with impact parameter $B$ dependence of the cross section, we find it natural to rewrite Eq. (2) in coordinate space, introducing the double distributions $D_{p, A}$ which are the Fourier conjugate of $G_{p, A}$ with respect to $\vec{\Delta}$. In such a representation, these distributions admit a probabilistic interpretation and represent the number density of parton pairs with longitudinal fractional momenta $x_{1}, x_{2}$, at a relative transverse distance $\vec{b}_{\perp}$, the latter being the Fourier conjugate to $\vec{\Delta}$.

In the impulse approximation for the nuclei, neglecting possible corrections to factorization due to the shadowing for large nuclei, and taking into account that $R_{A} \gg R_{p}$ for heavy nuclei, we can rewrite the cross section as $[14,25]$ 


$$
\begin{aligned}
\frac{d \sigma_{D P S}^{C D}}{d \Omega_{1} d \Omega_{2}}= & \frac{m}{2} \sum_{i, j, k, l} \sum_{N=p, n} \int d \vec{b}_{\perp} \int d^{2} B D_{p}^{i j}\left(x_{1}, x_{2} ; \vec{b}_{\perp}\right) D_{N}^{k l}\left(x_{3}, x_{4} ; \vec{b}_{\perp}\right) T_{N}(B) \frac{d \hat{\sigma}_{i k}^{C}}{d \Omega_{C}} \frac{d \hat{\sigma}_{j l}^{D}}{d \Omega_{D}}, \\
& +\frac{m}{2} \sum_{i, j, k, l} \sum_{N_{3}, N_{4}=p, n} \int d \vec{b}_{\perp} D_{p}^{i j}\left(x_{1}, x_{2} ; \vec{b}_{\perp}\right) \int d^{2} B f_{N_{3}}^{k}\left(x_{3}\right) f_{N_{4}}^{l}\left(x_{4}\right) T_{N_{3}}(B) T_{N_{4}}(B) \frac{d \hat{\sigma}_{i k}^{C}}{d \Omega_{C}} \frac{d \hat{\sigma}_{j l}^{D}}{d \Omega_{D}} .
\end{aligned}
$$

Here, $m=1$ if $C$ and $D$ are identical final states and $m=2$ otherwise, $i, j, k, l=\{q, \bar{q}, g\}$ are the parton species contributing to the final states $C(D)$. In Eq. (3) and in the following, $d \hat{\sigma}$ indicates the partonic cross section for producing the final state $C(D)$, differential in the relevant set of variables, $\Omega_{C}$ and $\Omega_{D}$, respectively. The functions $f^{i}$ appearing in Eq. (3) are single parton densities, and the subscript $N$ indicates nuclear parton distributions. The double parton distributions $D_{N}$ are the Fourier conjugate of ${ }_{2}$ GPDs of the nucleon bound in the nuclei.

The partonic cross sections and parton densities additionally depend on factorization and renormalization scales whose values are set to appropriate combination of the large scales occurring in final states $C$ and $D$.

The nuclear thickness function $T_{p, n}(B)$, mentioned in the Introduction and appearing in Eq. (3), is obtained by integrating the proton and neutron densities $\rho_{0}^{(p, n)}$ in the nucleus over the longitudinal component $z$

$$
T_{p, n}(B)=\int d z \rho^{(p, n)}(B, z)
$$

where we have defined $r$, the distance of a given nucleon from nucleus center, in terms of the impact parameter $B$ between the colliding proton and nucleus, $r=\sqrt{B^{2}+z^{2}}$. Following Ref. [36], for the ${ }^{208} P_{b}$ nucleus, the density of the proton and neutron is described by a Wood-Saxon distribution,

$$
\rho^{(p, n)}(r)=\frac{\rho_{0}^{(p, n)}}{1+e^{\left(r-R_{0}^{(p, n)}\right) / a_{(p, n)}} .}
$$

For the neutron density, we use $R_{0}^{n}=6.7 \mathrm{fm}$ and $a_{n}=$ $0.55 \mathrm{fm}$ [37]. For the proton density, we use $R_{0}^{p}=6.68 \mathrm{fm}$ and $a_{p}=0.447 \mathrm{fm} \mathrm{[38].} \mathrm{The} \rho_{0}^{(p, n)}$ parameters are fixed by requiring that the proton and neutron density, integrated over all distance $r$, are normalized to the number of the protons and neutrons in the lead nucleus, respectively.

As already anticipated, the DPS1 contribution, the first term in Eq. (3), stands for the contribution already at work in $p p$ collisions. It depends linearly on the nuclear thickness function $T$ and therefore scales as the number of nucleons in the nucleus, $A$.

The second term, the DPS2 contribution, contains in principle two-body nuclear distributions. We work here in the impulse approximation, neglecting short-range correlations in the nuclei since their contribution may change the results by several percent only [31]. The latter term is therefore proportional to the product of one-body nucleonic densities in the nucleus; i.e., it depends quadratically on $T$ and parametrically scales as $A^{4 / 3}$.

We shall work here in the mean field approximation for the nucleon. In such an approximation, double parton distributions have a factorized form,

$$
D_{p}^{i j}\left(x_{1}, x_{2}, \mu_{A}, \mu_{B}, \vec{b}_{\perp}\right) \simeq f_{p}^{i}\left(x_{1}, \mu_{A}\right) f_{p}^{j}\left(x_{2}, \mu_{B}\right) \mathcal{T}\left(\vec{b}_{\perp}\right),
$$

where the function $\mathcal{T}\left(\vec{b}_{\perp}\right)$ describes the probability of finding two partons at a relative transverse distance $\vec{b}_{\perp}$ in the nucleon and is normalized to unity. In such a simple approximation, this function does not depend on parton flavor and fractional momenta. Then, one may define the so-called effective cross section as

$$
\sigma_{\mathrm{eff}}^{-1}=\int d \vec{b}_{\perp}\left[\mathcal{T}\left(\vec{b}_{\perp}\right)\right]^{2}
$$

which controls the double parton interaction rate. Under all these approximations, the DPS cross section in $p A$ collision can be rewritten as

$$
\begin{aligned}
\frac{d \sigma_{D P S}^{C D}}{d \Omega_{1} d \Omega_{2}}= & \frac{m}{2} \sum_{i, j, k, l} \sum_{N=p, n} \sigma_{\text {eff }}^{-1} f_{p}^{i}\left(x_{1}\right) f_{p}^{j}\left(x_{2}\right) f_{N}^{k}\left(x_{3}\right) f_{N}^{l}\left(x_{4}\right) \frac{d \hat{\sigma}_{i k}^{C}}{d \Omega_{C}} \frac{d \hat{\sigma}_{j l}^{D}}{d \Omega_{D}} \int d^{2} B T_{N}(B), \\
& +\frac{m}{2} \sum_{i, j, k, l} \sum_{N_{3}, N_{4}=p, n} f_{p}^{i}\left(x_{1}\right) f_{p}^{j}\left(x_{2}\right) f_{N_{3}}^{k}\left(x_{3}\right) f_{N_{4}}^{l}\left(x_{4}\right) \frac{d \hat{\sigma}_{i k}^{C}}{d \Omega_{C}} \frac{d \hat{\sigma}_{j l}^{D}}{d \Omega_{D}} \int d^{2} B T_{N_{3}}(B) T_{N_{4}}(B) .
\end{aligned}
$$


TABLE I. Kinematic selection for the $4 j$ and $2 b 2 j$ final states adopted in experimental analyses and the corresponding values of extracted $\sigma_{\text {eff }}$.

\begin{tabular}{lccc}
\hline \hline Ref. & Selection & $\sigma_{\text {eff }}(m b)$ \\
\hline$[40]$ & $N_{\text {jets }} \geq 4, p_{T}^{j} \geq 20 \mathrm{GeV},\left|\eta_{j}\right| \leq 4.4$ and at least one having $p_{T} \geq 42.5 \mathrm{GeV}$ & $14.9_{-1.0}^{+1.2}(\mathrm{stat})_{-3.8}^{+5.1}(\mathrm{syst})$ \\
{$[41]$} & $N_{\text {jets }}=4:$ two jets with $p_{T} \geq 50 \mathrm{GeV}$ two jets with $p_{T} \geq 20 \mathrm{GeV},\left|\eta_{j}\right| \leq 4.7$ & $19.0_{-3.0}^{+4.6}[42]$ \\
{$[43]$} & Two light jets and two $b$ jets with $p_{T} \geq 20 \mathrm{GeV}\left|\eta_{b}\right| \leq 2.4,\left|\eta_{j}\right| \leq 4.7$ & $23.3_{-2.5}^{+3.3}[42]$ \\
\hline \hline
\end{tabular}

We find it important to state the key observation that leads to the second term of Eq. (3): namely, that the $b$ and $B$ integrals practically decouple since the nuclear density does not vary on subnuclear scale $[14,25,29]$. As a result, this term depends on ${ }_{2}$ GPDs integrated over transverse distance $b_{\perp}$, i.e., at $\vec{\Delta}=0$, for which we assume again mean field approximation:

$$
\int d \vec{b}_{\perp} D_{p}^{i j}\left(x_{1}, x_{2} ; \vec{b}_{\perp}\right) \simeq f_{p}^{i}\left(x_{1}\right) f_{p}^{j}\left(x_{2}\right) .
$$

After integration over $b_{\perp}$ in Eq. (7), $\sigma_{\text {eff }}$ will be the only nonperturbative parameter characterizing the DPS1 cross section. We use in our calculation $\sigma_{\text {eff }}$ values extracted from experimental analyses of DPS processes in $p p$ collisions. We neglect corrections due to longitudinal correlations in the nucleon [14] and any possible dependence of $\sigma_{\text {eff }}$ on energy [39]. For the considered final state, a number of experimental analyses have extracted $\sigma_{\text {eff }}$ values for $p p$ collisions at $\sqrt{s}=7 \mathrm{TeV}$ which are reported in Table I. In our numerical estimates, we use the average of those values, $\bar{\sigma}_{\text {eff }}=19 \mathrm{mb}$.

We close this section by specifying the kinematics and additional settings with which we evaluate Eq. (8). We consider proton lead collisions at a center-of-mass energy $\sqrt{s_{p N}}=8.16 \mathrm{TeV}$. Because of the different energies of the proton and lead beams $\left(E_{p}=6.5 \mathrm{TeV}\right.$ and $E_{P b}=$ $2.56 \mathrm{TeV}$ per nucleon), the resulting proton-nucleon center of mass is boosted with respect to the laboratory frame by $\Delta y=1 / 2 \ln E_{p} / E_{N}=0.465$ in the proton direction, assumed to be at positive rapidity. Therefore, jets rapidities, in this frame, are given by $y_{C M}=y_{l a b}-\Delta y$. All calculations are based on proton-nucleon center-of-mass rapidities.

All the relevant DPS and SPS cross sections contributing to the $4 j$ and $2 b 2 j$ final states have been calculated to leading order with ALPGEN [44]. Jet cross sections are obtained by identifying final state partons as jets, as appropriate for a leading-order calculations.

We use CTEQ6L1 leading-order free proton parton distributions [45]. Nuclear effects on the cross sections are estimated by using EPS09 nuclear parton distributions [46] in separate simulations. They are found to reduce the dijet cross sections less than $1 \%$ for $p_{T}^{j}>20 \mathrm{GeV}$ and are neglected. We also mention that dijet cross sections are, to very good accuracy, the same on target protons or neutrons, so no isospin corrections are applied.

\section{RESULTS: $\mathbf{4} \boldsymbol{j}$}

In this section, we present results for the inclusive production of, at least, four light jets. Among them, two (leading) jets are requested to have $p_{T}^{j_{1}, j_{2}}>50 \mathrm{GeV}$. Two (subleading) jets are requested to have $p_{T}^{j_{3}, j_{4}}>20 \mathrm{GeV}$. All jets are required to have $\left|y_{j}^{l a b}\right|<4$.7. Different cuts on the leading and subleading jets are enforced to facilitate the pairing for the DPS selection. For both the DPS and the SPS mechanisms, we require the interparton distance in the $\eta-\phi$ plane

$$
\Delta R_{i j}=\sqrt{\left(\eta_{i}-\eta_{j}\right)^{2}+\left(\phi_{i}-\phi_{j}\right)^{2}}
$$

to be $\Delta R_{i j}>0.7$, where $i$ and $j$ stand for a generic light jets $(i, j=1 \ldots 4, i \neq j)$.

In the DPS cross section given by Eq. (8), we set the symmetry factor $m=2$ when the subleading jets have $20<p_{T}^{j_{3}, j_{4}}<50 \mathrm{GeV}$ and $m=1$ if $p_{T}^{j_{3}, j_{4}}>50 \mathrm{GeV}$ (the leading jets in both cases have $p_{T}^{j_{1}, j_{2}}>50 \mathrm{GeV}$ ).

The factorization and renormalization scales are fixed to $\mu_{F}=\mu_{R}=\sqrt{\sum_{j}^{N \text { jet }} p_{T, j}^{2}}$, where $N_{\text {jet }}=2$ in DPS and $N_{\text {jet }}=4$ in SPS. All the calculations are performed with ALPGEN [44].

We report in Table II the various contributions to the $4 j$ fiducial cross section for three different transverse momentum cuts on the subleading jets. In the last three columns, we report the ratio between the four jets (SPS + DPS) over two jets (with $p_{T}>50 \mathrm{GeV}$ ) cross section, the DPS1 fraction $f_{D P S 1}$ calculated as the DPS1 over (DPS1+SPS) cross section (for easy comparison to $p p$ collisions), and the DPS2 fraction $f_{D P S 2}$, calculated as the DPS2 over (DPS1 + DPS2 + SPS) cross section. In general, we observe a large contribution from DPS2, which reaches $27 \%$ of the four-jet cross section for $p_{T}^{j_{3}, j_{4}}>20 \mathrm{GeV}$. We present in the left panel of Fig. 2 the various contributions to the cross sections differential in $B$ and in the right panel the expected number of events assuming $\int \mathcal{L} \mathrm{dt}=0.1 \mathrm{pb}^{-1}$, a value in line with data recorded in $2016 p A$ runs. Exploiting the different dependence on $T$ of the various contributions, we may use the strategy put forward in Ref. [31] to separate the DPS2 contribution. For this purpose, we evaluate the number of events integrating Eq. (1) in the $i$ bin specified by the binedge values $T_{i}$ and $T_{i+1}$, 
TABLE II. Predictions for $4 j$ DPS and SPS cross sections in $p A$ collisions in fiducial phase space, for different cuts on jets transverse momenta.

\begin{tabular}{lrrrrrrr}
\hline \hline & DPS1 & DPS2 & \multicolumn{1}{c}{ SPS } & Sum & $\sigma(4 j) / \sigma(2 j)$ & $f_{D P S 1}$ & $f_{D P S 2}$ \\
\cline { 2 - 8 } $4 j$ & $(\mu \mathrm{b})$ & $(\mu \mathrm{b})$ & \multicolumn{1}{c}{$(\mu \mathrm{b})$} & \multicolumn{1}{c}{$(\mu \mathrm{b})$} & & & \\
\hline$p_{T}^{j_{3}, j_{4}}>20 \mathrm{GeV}$ & 26.0 & 72.2 & 170.9 & 269.2 & 0.15 & 0.13 & 0.27 \\
$p_{T}^{j_{3}, j_{4}}>25 \mathrm{GeV}$ & 10.8 & 30.2 & 92.9 & 133.9 & 0.07 & 0.10 & 0.22 \\
$p_{T}^{j_{3}, j_{4}}>30 \mathrm{GeV}$ & 5.1 & 14.3 & 51.4 & 70.9 & 0.04 & 0.09 & 0.20 \\
\hline \hline
\end{tabular}

$$
\begin{aligned}
N_{e v}\left(T_{i}, T_{i+1}\right)= & \int d^{2} B \frac{d^{2} \sigma_{p A}}{d^{2} B} \Theta\left(T_{A}(B)-T_{i}\right) \\
& \times \Theta\left(T_{i+1}-T_{A}(B)\right),
\end{aligned}
$$

and then we consider the ratio $R_{4 j}$ between the total number (DPS + SPS) of four-jet events over those for dijet production (with $p_{T}>50 \mathrm{GeV}$ ) as a function of $T_{A}(B)$ :

$$
R_{4 j}\left(T_{i}, T_{i+1}\right)=N_{4 j}\left(T_{i}, T_{i+1}\right) / N_{2 j}\left(T_{i}, T_{i+1}\right) .
$$

In such a ratio, $N_{2 j}$ is linear in $T_{A}(B)$, as well as the SPS background, and the DPS1 mechanisms, which both contribute to $N_{4 j}$. In the absence of the quadratic DPS2 contribution, such a ratio would be a constant. Its presence, on the other hand, will induce a linear increase of the ratio as a function of $T$, and the size of the DPS2 contribution will determine its slope.

The resulting distribution is presented in the left panel of Fig. 3 for different values of jet transverse momenta cutoff and integrated in bins of $T$, chosen to evenly distribute the number of events. The rise of the slope is

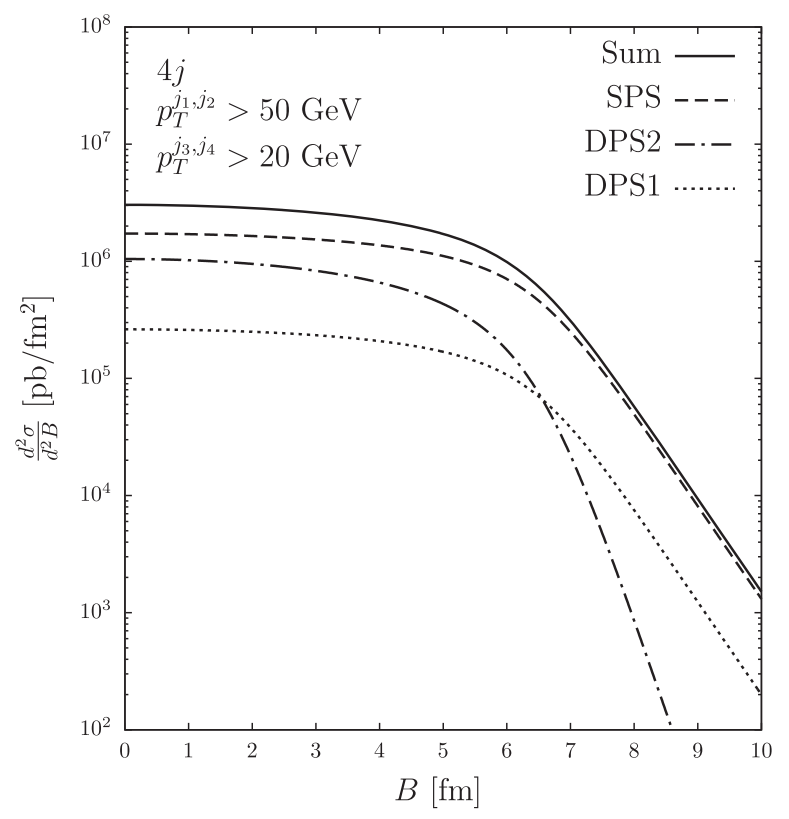

related to the fast rise of the dijet cross sections entering the DPS2 estimation as the cuts on jet transverse momenta are decreased. Our calculations were done to the leading order (LO) in strong coupling. Therefore, it is natural to ask about the stability of the ratio in Eq. (12). The role of higher-order corrections for the $4 j$ final state has been investigated in a number of papers, and corrections have been found to be large $[47,48]$. To partially overcome this problem, we form the double ratio

$$
R_{4 j}^{0}\left(T_{i}, T_{i+1}\right)=\frac{N_{4 j}\left(T_{i}, T_{i+1}\right)}{N_{2 j}\left(T_{i}, T_{i+1}\right)}\left(\frac{N_{4 j}\left(T_{0}, T_{1}\right)}{N_{2 j}\left(T_{0}, T_{1}\right)}\right)^{-1} ;
$$

i.e., we normalize it to the first bin with $T_{0}=0 \mathrm{fm}^{-2}$ and $T_{1}=1 \mathrm{fm}^{-2}$. The resulting distribution is presented in the right panel of Fig. 3. Assuming that statistical errors follow a Poissonian distribution, the associated error is derived from the expected number of events. Our results indicate that, within these errors estimates, the departure from a constant behavior can be unambiguously appreciated and the DPS2 contribution can be disentangled

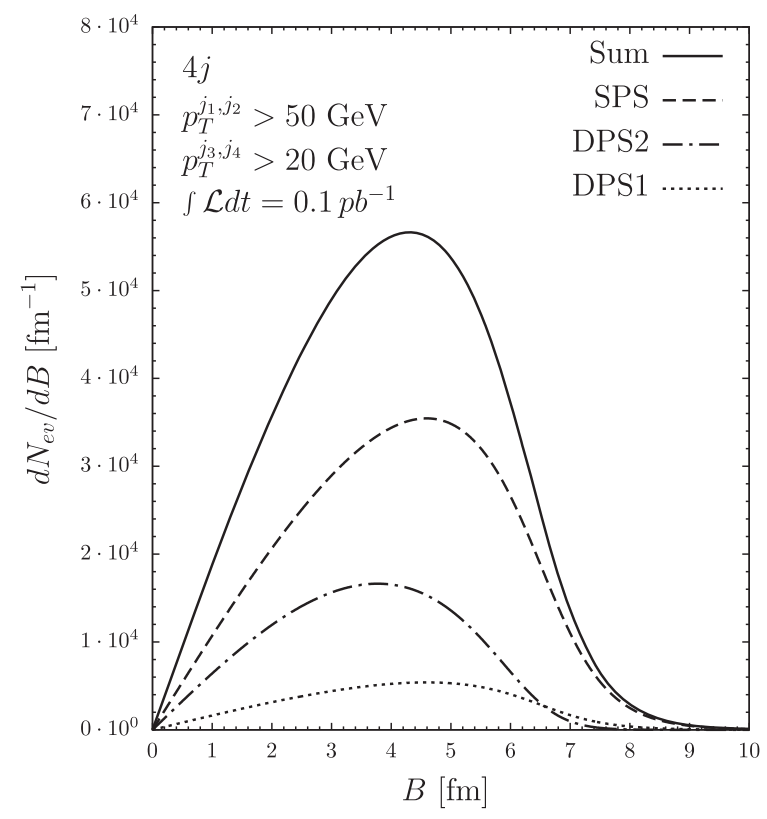

FIG. 2. Differential cross section as a function of $B$ for the various contributions to the $4 j$ final state (left). Expected number of events for the various contributions assuming $\int \mathcal{L} \mathrm{dt}=0.1 \mathrm{pb}^{-1}$ (right). 

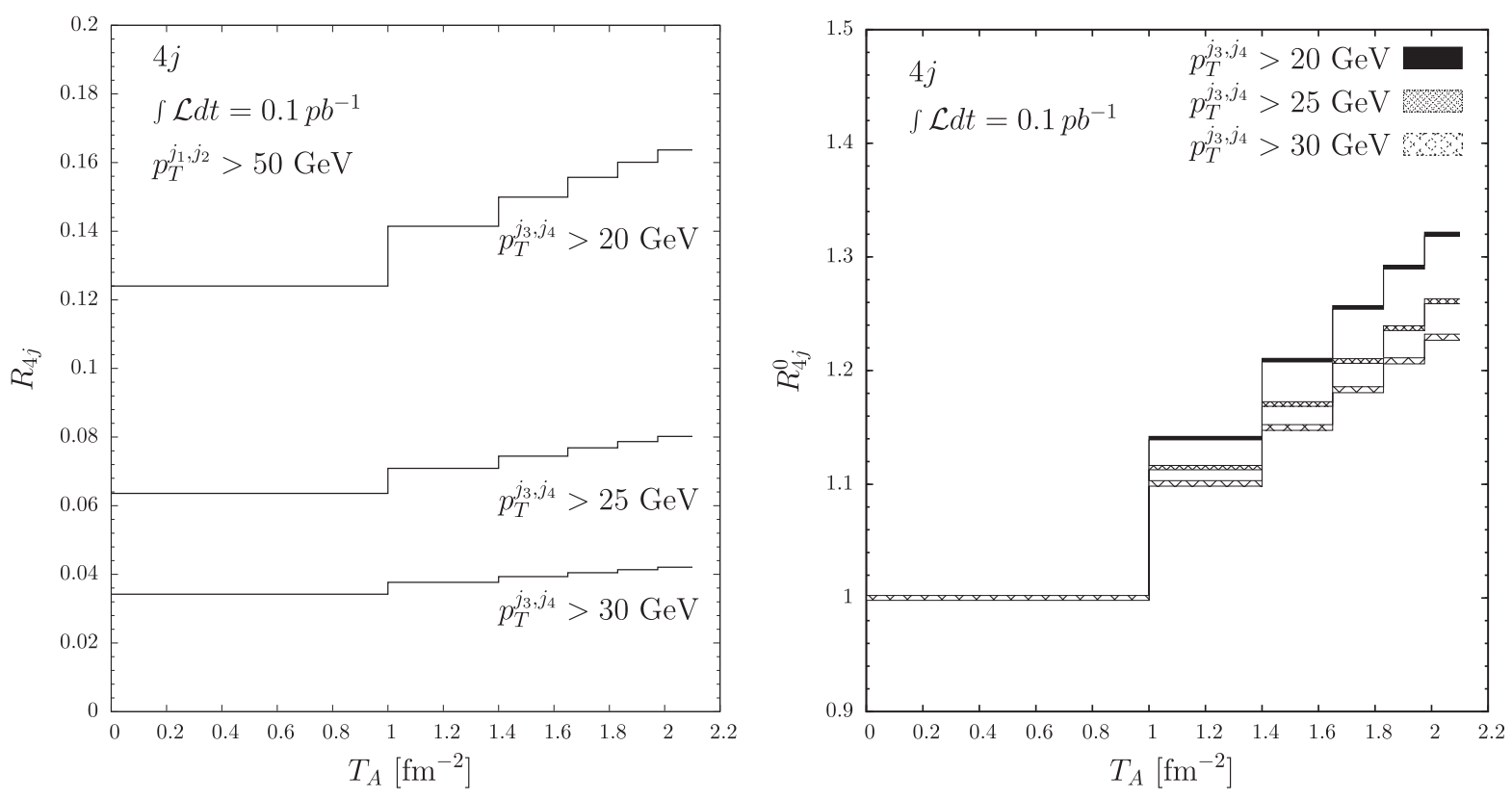

FIG. 3. The ratio in Eq. (12) (left) and double ratio in Eq. (13) (right) integrated in bins of $T_{A}(B)$. Predictions are shown for three different cuts on jet transverse momenta.

already from data of $2016 p A$ runs, modulo the experimental issues in studying the most peripheral events.

\section{RESULTS: $2 b 2 j$}

We consider in this section a special class of the former process in which the second scattering produces a $b \bar{b}$ heavy-quark pair. Experimental results for this final state are reported in Ref. [43]. Light and heavy quark jets are all requested to have $p_{T}>20 \mathrm{GeV}$. Additionally, light jets are requested to have $\left|\eta_{j}^{l a b}\right|<4.7$, and heavy quark jets are requested to have $\left|\eta_{b}^{l a b}\right|<2.4$. For this final state, the symmetry factor in the DPS cross sections is set to $m=2$. The additional heavy quark tagging facilitates the pairing in the DPS selection. For both DPS and SPS mechanisms, we require $\Delta R_{i j}>0.7$ where both indices run over light and heavy quark jets. The factorization and renormalization scales are fixed to $\mu_{F}=\mu_{R}=\sqrt{\sum_{j}^{N \text { jet }} m_{T, j}^{2}}$, where $N_{\text {jet }}=2$ in DPS and $N_{\text {jet }}=4$ in SPS, and $m_{T, j}=\sqrt{m_{j}^{2}+p_{T, j}^{2}}$ is the transverse mass of jet $j$. All the calculations are performed with ALPGEN [44].

We report in Table III the various contributions to the $2 b 2 j$ fiducial cross section for three different transverse momentum cuts on the jets. In general, we observe a large contribution from DPS2, which reaches $29 \%$ for $p_{T}>20 \mathrm{GeV}$. We present in the left panel of Fig. 4 the various contributions to the cross sections differential in $B$ and in the right panel the expected number of events assuming $\int \mathcal{L} \mathrm{dt}=0.1 \mathrm{pb}^{-1}$. As in the previous section, we consider the ratio $R_{2 b 2 j}$ between the total number of $2 b 2 j$ events (DPS + SPS) over those for dijet production as a function of $T_{A}(B)$ :

$$
R_{2 b 2 j}\left(T_{i}, T_{i+1}\right)=N_{2 b 2 j}\left(T_{i}, T_{i+1}\right) / N_{2 j}\left(T_{i}, T_{i+1}\right) .
$$

The resulting distribution is presented in the left panel of Fig. 5 for different values of jet transverse momenta cutoff and integrated in bins of $T$. The rise of the slope is related to fast rise of the dijet cross sections entering the DPS2

TABLE III. Predictions for $2 b 2 j$ DPS and SPS cross sections in $p A$ collisions in fiducial phase space for different cuts on jets transverse momenta.

\begin{tabular}{lccccccc}
\hline \hline & DPS1 & DPS2 & SPS & Sum & $\sigma(2 b 2 j) / \sigma(2 j)$ & $f_{D P S 1}$ & $f_{D P S 2}$ \\
\cline { 2 - 8 } $2 b 2 j$ & $(\mu \mathrm{b})$ & $(\mu \mathrm{b})$ & $(\mu \mathrm{b})$ & $(\mu \mathrm{b})$ & $\times 10^{-4}$ & & \\
\hline$p_{T}^{b, j}>20 \mathrm{GeV}$ & 2.2 & 6.2 & 13.0 & 21.4 & 3.0 & 0.15 & 0.29 \\
$p_{T}^{b, j}>25 \mathrm{GeV}$ & 0.4 & 1.2 & 4.7 & 6.4 & 2.1 & 0.09 & 0.19 \\
$p_{T}^{b, j}>30 \mathrm{GeV}$ & 0.1 & 0.3 & 1.9 & 2.3 & 1.6 & 0.06 & 0.13 \\
\hline \hline
\end{tabular}



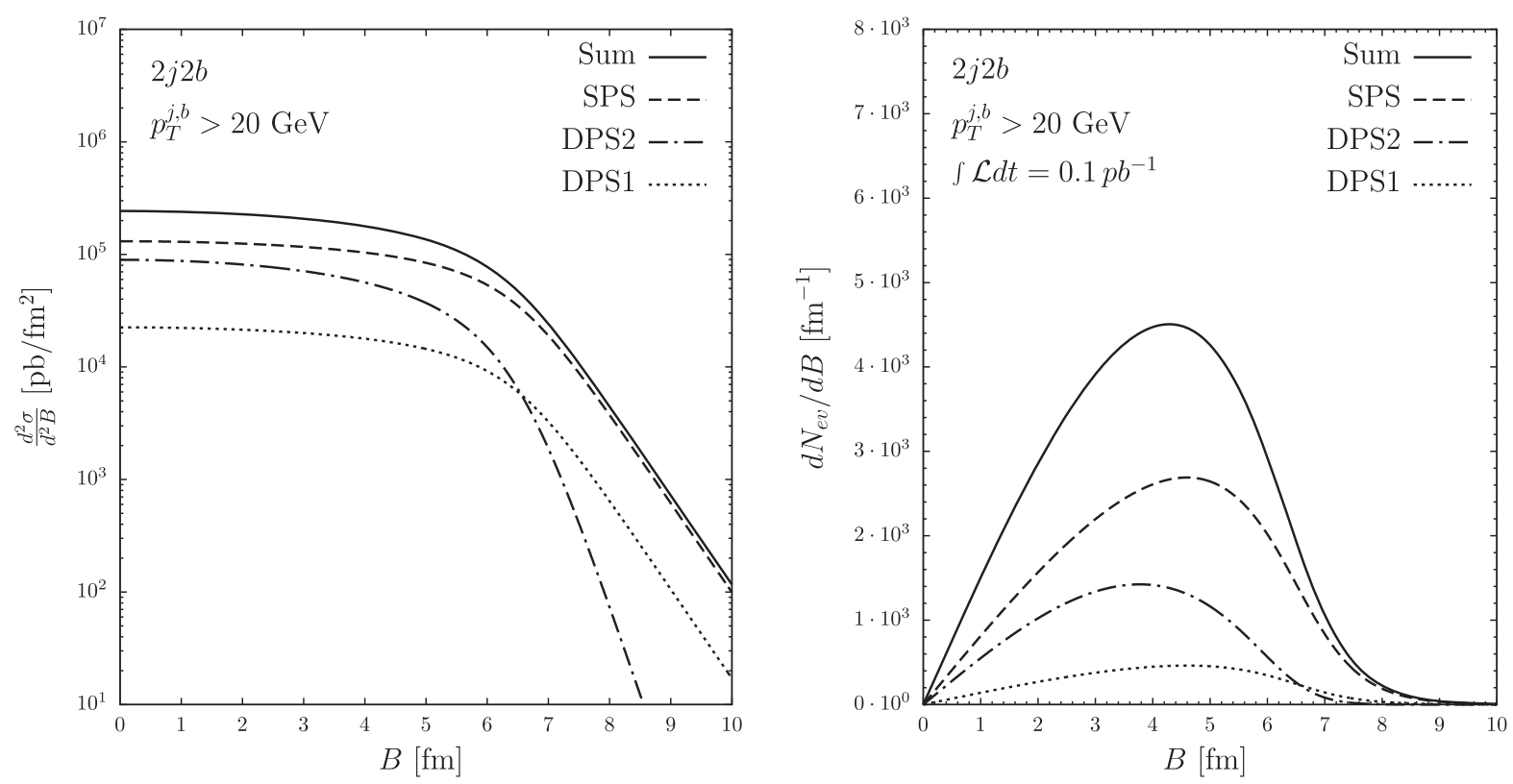

FIG. 4. Differential cross section as a function of $B$ for the various contributions to the $2 b 2 j$ final state (left). Expected number of events for the various contributions assuming $\int \mathcal{L} \mathrm{dt}=0.1 \mathrm{pb}^{-1}$ (right).

estimation as the cuts on jet transverse momenta are decreased. As shown in Table III of Ref. [43], the comparison of various theoretical predictions with $2 b 2 j$ data reveal substantial agreement with next-to-leadingorder predictions, but LO prediction suffers from large higher-order corrections. Such results are confirmed also by the ALPGEN prediction, which returns a cross section 0.6 times smaller than data [43]. To partly mitigate these effects, we form the double ratio

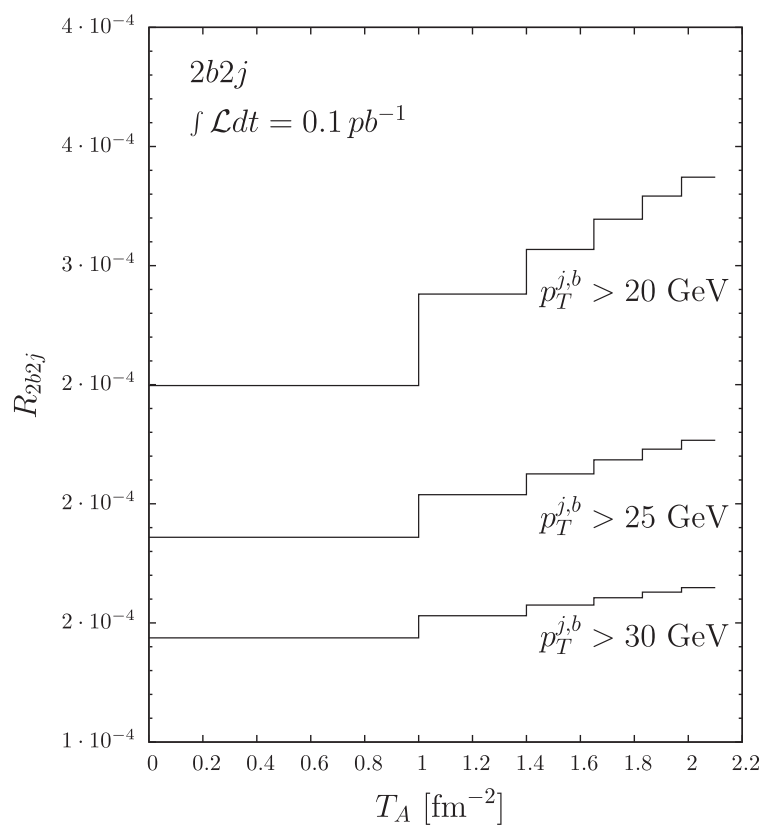

$$
R_{2 b 2 j}^{0}\left(T_{i}, T_{i+1}\right)=\frac{N_{2 b 2 j}\left(T_{i}, T_{i+1}\right)}{N_{2 j}\left(T_{i}, T_{i+1}\right)}\left(\frac{N_{2 b 2 j}\left(T_{0}, T_{1}\right)}{N_{2 j}\left(T_{0}, T_{1}\right)}\right)^{-1}
$$

i.e., we normalize it to the first bin $(0<T<1)$. The resulting distribution is presented in the right panel of Fig. 5. The associated error is calculated from the expected number of events, assuming a Poissonian distribution for statistical errors.

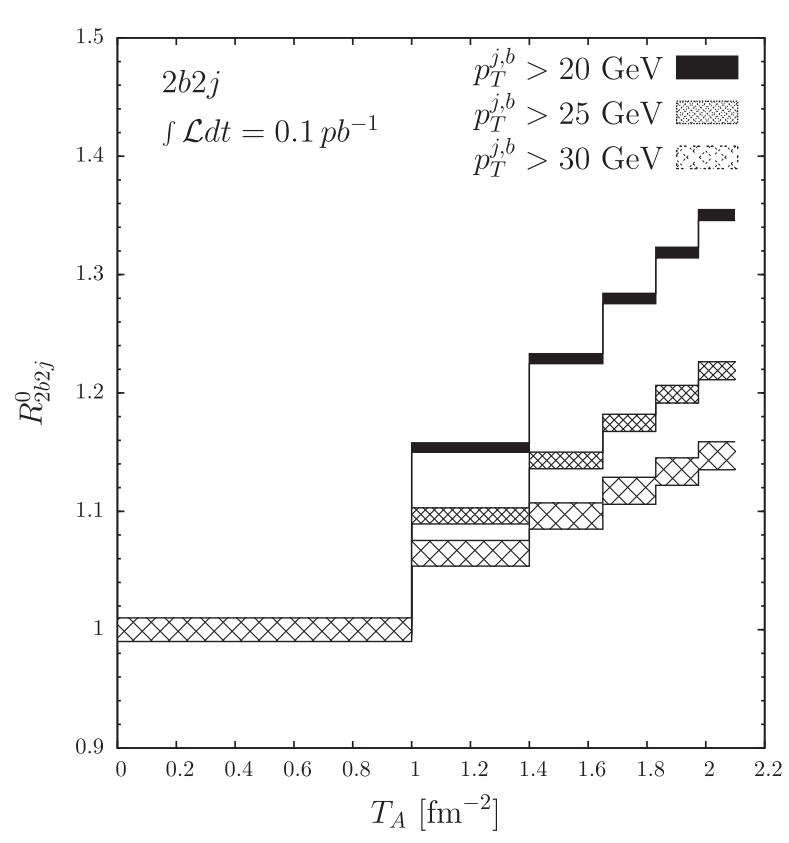

FIG. 5. The ratio in Eq. (14) (left panel) and double ratio in Eq. (15) (right panel) integrated in bins of $T_{A}(B)$. Predictions are shown for three different cuts on jet transverse momenta. 
Our results indicate that, although with lesser significance with respect to the four-jet case, the departure from a constant behavior can be unambiguously observed also in this final state. As already observed in the $4 j$ case, lowering the cut on the jet transverse momenta increases the sensitivity to a nonconstant behavior of $R_{2 b 2 j}^{0}$.

\section{CONCLUSIONS}

In this paper, we have calculated DPS cross sections for double dijet final states produced in $p A$ collisions at the LHC as well as the corresponding SPS backgrounds. Relying on the experimental capabilities to correlate centrality with impact parameter $B$ of the protonnucleus collision, we have presented a strategy to extract the so-called DPS2 contributions, pertinent to $p A$ collisions. In this respect, the $4 j$ and $2 b 2 j$ final states have large enough cross sections to allow the use of the method [31] to disentangle leading twist + DPS1 contributions from the DPS2 contribution, which is the main interest of this paper, already with data accumulated in the $2016 p A$ run.

\section{ACKNOWLEDGMENTS}

The authors would like to thank M. Strikman for reading the manuscript and for many useful discussions. We also thank A. Milov for many useful comments. The work was supported by Israel Science Foundation under Grant No. 2025311. The diagram in this paper has been drawn with JAXODRAW package version 2.0 [49].
[1] N. Paver and D. Treleani, Nuovo Cimento A 70, 215 (1982).

[2] N. Paver and D. Treleani, Phys. Lett. 146B, 252 (1984).

[3] M. Mekhfi, Phys. Rev. D 32, 2371 (1985).

[4] J. R. Gaunt and W. J. Stirling, J. High Energy Phys. 03 (2010) 005.

[5] B. Blok, Yu. Dokshitzer, L. Frankfurt, and M. Strikman, Phys. Rev. D 83, 071501 (2011).

[6] M. Diehl, Proc. Sci., DIS2010 (2010) 223 [arXiv:1007 $.5477]$.

[7] J. R. Gaunt and W. J. Stirling, J. High Energy Phys. 06 (2011) 048.

[8] B. Blok, Yu. Dokshitser, L. Frankfurt, and M. Strikman, Eur. Phys. J. C 72, 1963 (2012).

[9] M. Diehl, D. Ostermeier, and A. Schafer, J. High Energy Phys. 03 (2012) 089.

[10] B. Blok, Yu. Dokshitser, L. Frankfurt, and M. Strikman, arXiv:1206.5594v1.

[11] B. Blok, Yu. Dokshitzer, L. Frankfurt, and M. Strikman, Eur. Phys. J. C 74, 2926 (2014).

[12] M. Diehl, J. R. Gaunt, and K. Schönwald, J. High Energy Phys. 06 (2017) 083.

[13] A. V. Manohar and W. J. Waalewijn, Phys. Rev. D 85, 114009 (2012).

[14] B. Blok, M. Strikman, and U. A. Wiedemann, Eur. Phys. J. C 73, 2433 (2013).

[15] Advanced Series on Directions in High Energy Physics, edited by P. Bartalini and J. Gaunt (World Scientific, Singapore, 2018), Vol. 29, p. 2019.

[16] I. M. Belyaev, 7th International Workshop on Multiple Partonic Interactions at the LHC", Trieste, Italy (2015).

[17] R. Aaij et al. (LHCb Collaboration), J. High Energy Phys. 06 (2012) 141; 03 (2014) 108(A).

[18] R. Aaij et al. (LHCb Collaboration), Nucl. Phys. B871, 1 (2013).

[19] R. Aaij et al. (LHCb Collaboration), J. High Energy Phys. 07 (2016) 052.
[20] A. M. Sirunyan et al. (CMS Collaboration), Eur. Phys. J. C 80, 41 (2020).

[21] T. Sjöstrand et al., Comput. Phys. Commun. 191, 159 (2015).

[22] M. Bahr et al., Eur. Phys. J. C 58, 639 (2008).

[23] G. Calucci and D. Treleani, Phys. Rev. D 60, 054023 (1999).

[24] M. Rinaldi and F. A. Ceccopieri, Phys. Rev. D 97, 071501 (2018).

[25] M. Strikman and D. Treleani, Phys. Rev. Lett. 88, 031801 (2002).

[26] I. Helenius and H. Paukkunen, Phys. Lett. B 800, 135084 (2020).

[27] D. d'Enterria and A. Snigirev, Adv. Ser. Dir. High Energy Phys. 29, 159 (2018).

[28] E. Cattaruzza, A. Del Fabbro, and D. Treleani, Phys. Rev. D 70, 034022 (2004).

[29] S. Salvini, D. Treleani, and G. Calucci, Phys. Rev. D 89, 016020 (2014).

[30] O. Fedkevych and L. Lönnblad, arXiv:1912.08733.

[31] M. Alvioli, M. Azarkin, B. Blok, and M. Strikman, Eur. Phys. J. C 79, 482 (2019).

[32] B. Blok and F. A. Ceccopieri, Eur. Phys. J. C 80, 278 (2020).

[33] G. Aad et al. (ATLAS Collaboration), Eur. Phys. J. C 76, 199 (2016).

[34] G. Aad et al. (ATLAS Collaboration), Phys. Lett. B 756, 10 (2016).

[35] G. Aad et al. (ATLAS Collaboration), Phys. Lett. B 763, 313 (2016).

[36] M. Alvioli and M. Strikman, Phys. Rev. C 100, 024912 (2019).

[37] C. M. Tarbert et al., Phys. Rev. Lett. 112, 242502 (2014).

[38] M. Warda, X. Vinas, X. Roca-Maza, and M. Centelles, Phys. Rev. C 81, 054309 (2010).

[39] B. Blok and M. Strikman, Phys. Lett. B 772, 219 (2017).

[40] M. Aaboud et al. (ATLAS Collaboration), J. High Energy Phys. 11 (2016) 110. 
[41] S. Chatrchyan et al. (CMS Collaboration), Phys. Rev. D 89, 092010 (2014).

[42] P. Gunnellini, https://doi.org/10.3204/DESY-THESIS-2015010.

[43] V. Khachatryan et al. (CMS Collaboration), Phys. Rev. D 94, 112005 (2016).

[44] M. L. Mangano, M. Moretti, F. Piccinini, R. Pittau, and A. D. Polosa, J. High Energy Phys. 07 (2003) 001.
[45] J. Pumplin et al., J. High Energy Phys. 07 (2002) 012.

[46] K. J. Eskola, H. Paukkunen, and C. A. Salgado, J. High Energy Phys. 04 (2009) 065.

[47] Z. Bern et al., Phys. Rev. Lett. 109, 042001 (2012).

[48] S. Badger, B. Biedermann, P. Uwer, and V. Yundin, Phys. Lett. B 718, 965 (2013).

[49] D. Binosi, J. Collins, C. Kaufhold, and L. Theussl, Comput. Phys. Commun. 180, 1709 (2009). 